\title{
Resposta à flexão e análise de tenacidade de argamassas reforçadas com fibra de Curauá
}

\author{
Bending answer and toughness analysis \\ of mortar reinforced with \\ Curauá fibers
}

\author{
Maurílio Gomes Pimentel ${ }^{1}$, João Pedro das Chagas Borges ${ }^{1}$, \\ Marcelo de Souza Picanço ${ }^{1}$, Khosrow Ghavami ${ }^{2}$
}

\footnotetext{
${ }^{1}$ Laboratório de Engenharia Civil - LEC/PPGEC/UFPA CEP: 66075 - 110, Belém, PA e-mail: mauriliogpimentel@gmail.com, joaopedrocborges@gmail.com, marcelosp@ufpa.br

${ }^{2}$ Departamento de Engenharia Civil da Pontifícia Universidade Católica do Rio de Janeiro - PUC CEP: 22451-900 e-mail: ghavami@civ.puc-rio.br
}

\section{RESUMO}

O desenvolvimento e comercialização de materiais compósitos produzidos a partir de fibras naturais são considerados extremamente importante, uma vez que essas fibras reduzirão a dependência dos materiais produzidos com recursos não renováveis. Dentre essas fibras naturais destaca-se a fibra do curauá, sua utilização na produção de compósitos melhora de forma notável as propriedades do conjunto fibra matriz, logo o presente artigo objetiva estudar as propriedades mecânicas de compósitos reforçados com fibra de curauá, em especial sua resistência à flexão e tenacidade. Para isso foram confeccionadas cinco famílias de argamassas, uma de referência sem utilização da fibra e as outras quatro reforçada com fibra variando o comprimento da fibra e sua fração volumétrica. Verificou-se que a fibra do curauá ao ser adicionada em matriz cimentícia melhora suas propriedades mecânicas comparada a um compósito não reforçado com fibra, sua deformação, resistência à flexão e tenacidade são melhoradas.

Palavras-chave: comportamento mecânico, fibras naturais, materiais não convencionais, compósitos cimentícios, fibra de curauá.

\begin{abstract}
The composites material produced from natural fibers development and commercialization are considered extremely important because these fibers will reduce the material produced with non-renewable resources dependence. Among these fibers, there is the Curauá fiber, its use in composites production improves the ensemble matrice fiber properties, then this article aims to study the composites reinforced with Curauá fibers mechanical properties, especially its bending resistance and toughness. For achieve those objectives, it was produced five mortar groups, one for reference, without the fiber utilization, and another four groups with fiber reinforcement varying the fiber length and its volume fraction. It was noted that Curauá fiber, when added to cement matrice, improves its mechanical properties compared to a composite without the fiber reinforcement, its deflection, bending resistance and toughness are improved.
\end{abstract}

Keywords: Mechanical behavior, Natural fibers, Non-conventional material, cement composites, Curauá fibers.

\section{INTRODUÇÃO}

O interesse no mundo relacionado a uso de materiais naturais renováveis para substituir materiais sintéticos vem crescendo. As fibras vegetais naturais parecem oferecer uma grande oportunidade a esse respeito, dentre essas fibras destaca-se a fibra do curauá, obtida por meio da extração das folhas da Ananas erectifolius, que normalmente é chamada de curauá [3], cujo seu cultivo é feito principalmente na região amazônica. Sua produção no Brasil no ano de 2003 chegou a 150 toneladas [7] e isso vem aumentando com os anos. 
A fibra do curauá possui propriedades únicas como baixa densidade, alta resistência, uma estrutura fina e um alto teor de celulose [1], além de possuir tato relativamente macio [2]. Suas propriedades mecânicas mostram-se superior comparadas as fibras como bambu, palmeira e sisal [4] além de se mostrar como um material promissor como agente de reforço em compósito de matriz polimérica e cimentícia [4], [5], [6].

A utilização de fibras em compósitos de matriz cimentícia tende a aumentar a resistência à compressão do compósito [8]. A atuação das mesmas é marcante depois de atingida a carga de pico, que corresponde ao início da fissuração da argamassa. Assim, ao invés da ruptura brusca apresentada pela matriz plena, o compósito continua a suportar carga, embora em níveis inferiores à carga de pico, apresentando grande deformação [9].

Quando uma viga retangular de material elástico é carregada em flexão, as tensões deformações longitudinais em uma dada seção transversal variam de forma linear da superfície em compressão, para atingir um máximo de tração na superfície oposta [10]. A tensão de ruptura, calculada a partir do momento de flexão, assumindo-se um comportamento elástico, é conhecida como módulo de ruptura e é uma medida da resistência à tração do material. Na prática, mesmo para um material elástico, o módulo de ruptura é geralmente maior que a resistência à tração, pois um volume menor do corpo de prova é tencionado e tensões de flexão, que podem surgir em um ensaio de tração pelo desalinhamento das garras, são eliminados. No entanto, a teoria convencional das vigas é inadequada para compósitos reforçados por fibras, pois a curva tensão $\mathrm{x}$ deformação pós fissuração no lado tracionado é diferente observada em compressão.

Mesmo se o compósito for considerado como um material idealmente elástico, antes da fissuração da matriz, a teoria da flexão não pode ser considerada para o comportamento em flexão além deste ponto [10]. Quando o limite elástico na flexão, em um material elástico perfeito, é alcançado, haverá ruptura. No entanto, o elasto plástico pode continuar suportando cargas adicionais. Tal fato é acompanhado por modificações na distribuição das tensões, com a linha neutra movendo-se para cima e a distribuição das tensões de tração se tornando retangular. Como resultado, a curva carga-deflexão no material elasto plástico continuará a ascender além do limite elástico. Assim, a ductilidade associada ao comportamento pseudo plástico leva a um aumento da capacidade de carga do material idealmente plástico, mesmo que na resistência à tração não seja maior que aquela do material idealmente elástico.

Além da determinação da resistência sob flexão, a partir do diagrama carga deflexão é possível determinar a tenacidade dos compósitos. A tenacidade é uma importante característica para os materiais compósitos constituídos de fibras vegetais, sendo geralmente aceito que um dos principais papéis desempenhados pelas fibras é prover tenacidade às matrizes frágeis [11]. Muitos ensaios podem ser aplicados para caracterização da tenacidade dos compósitos tais como tração, compressão, impacto e flexão [4]. O ensaio de flexão é mais utilizado, pois o mesmo apresenta mais verdadeiramente as condições de muitas situações práticas e é mais fácil de ser realizado que o ensaio de tração, por exemplo.

Com a finalidade de usar a fibra do curauá como agente de reforço em compósitos cimentícios, no presente trabalho objetiva-se estudar o comportamento á flexão bem como a análise da tenacidade em compósitos cimentícios reforçados com fibra de curauá.

\section{MATERIAIS E MÉTODOS}

O programa experimental consistiu em uma matriz de argamassa sem reforço das fibras de curauá, tomada como referência, e uma matriz de argamassa reforçada com fibras de curauá, em dois teores e comprimentos de fibra. As argamassas reforçadas com fibras foram produzidas nas mesmas condições e moldadas em corpos de prova prismáticos. Para avaliar o comportamento a flexão dos compósitos, foram utilizados procedimentos propostos pela norma ASTM C1609, Standard test method for flexural performance of FiberReinforced concret (Using Beam with third-point loading) [13], que determina o uso de sistema fechado com controle de velocidade de deslocamento, da norma NBN B 15-238, Tests on fibre reinforced concret - Bending test on prismatic sample [14], que determina índices de tenacidade através das curvas carga versus deflexão e do método JSCE -SF4-Method of tests for flexural strength and flexural toughness of steel fiber reinforced concrete [15] que é amplamente utilizado no Brasil e um dos métodos mais usados para dimensionamento de matrizes reforçadas com fibras.

\subsection{Materiais utilizados}

Os materiais utilizados nas argamassas foram cimento Portland CP II - F - 32, agua potável e areia natural. A areia teve a massa unitária determinada de acordo com norma NBR NM 45, Agregados - Determinação da massa unitária e índices de vazios [12], resultando em $1,47 \mathrm{~g} / \mathrm{cm}^{3}$. A massa específica foi determinada de acordo com a norma NBR NM 52, Agregado miúdo - determinação da massa específica e da massa específi- 
ca aparente [16], resultando em 2,63 g/ $\mathrm{cm}^{3}$. Já a composição granulométrica foi determinada de acordo com a norma NBR NM 248, Agregado miúdo - determinação da composição granulométrica [17], resultando em um módulo de finura de 2,69 mm e um diâmetro máximo de 4,8 mm.

Para a caracterização das fibras, inicialmente aferiu-se o diâmetro das fibras através de dois métodos, o primeiro foi com o uso de um paquímetro de precisão de 0,01 mm em três regiões da fibra (dois pontos próximos às extremidades e um próximo ao centro), sendo usados 30 exemplares de fibra com $50 \mathrm{~mm}$ de comprimento. Com as leituras obtidas calculou - se a média aritmética resultando em um diâmetro médio de 0,092 mm. O segundo, com maior precisão, foi através da projeção de luz com o uso de um equipamento projetor de perfil, com magnificação de até 100 vezes, usando 30 exemplares de fibras com $50 \mathrm{~mm}$ de comprimento e calculando-se a média aritmética, resultando em um diâmetro médio de 0,127 $\mathrm{mm}$. Tal diferença explica-se pelo fato de o método do paquímetro ser menos preciso em relação ao segundo método, devido a grande variabilidade de diâmetros ao longo da fibra e ao possível esmagamento da fibra no momento de sua aferição.

Em seguida, aferiu-se a massa específica das fibras através do frasco de Chapman, as fibras foram cortadas com comprimentos médios de $30 \mathrm{~mm}$ para facilitar a introdução das mesmas nos frascos e depois foram secas em estufas até a constância de massa (Até que duas pesagens consecutivas espaçadas de $2 \mathrm{~h}$ não apresentassem variação de massa). Inseriu-se as $50 \mathrm{mg}$ de fibras no frasco e foram deixadas imersas por $24 \mathrm{~h}$ antes de ser efetuada a leitura do nível de água no gargalo do frasco para que os vazios das fibras pudessem ser ocupados pela água e o calculo da massa específico foi feito pela equação 1 , em que $\mathrm{m}_{\mathrm{fi}}$ é a massa da amostra de fibras secas, L é a leitura no gargalo do frasco e subtrai-se 200 de L pois é a leitura inicial no frasco sem as fibras, obtendo-se o valor $\mu$ de massa específica de $1,37 \mathrm{~g} / \mathrm{cm}^{3}$.

$$
\mu=\frac{m_{f i}}{L-200}
$$

Determinou-se a absorção de água secando as fibras até a constância de massa e colocando-as em imersão em água e aferindo a massa em intervalos de 5 min, 30 min, 1 h, 2 h e em seguida em intervalos de $24 \mathrm{~h}$ até o sexto dia e então em intervalos de 48 h até o ponto de saturação (ponto em que não há variação de massa em duas pesagens consecutivas), utilizou-se a equação 2 para se calcular a absorção de água das fibras onde A é o percentual de absorção de água das fibras, $\mathrm{P}_{\mathrm{ht}}$ é o peso úmido da fibra no tempo t, $\mathrm{P}_{\mathrm{e}}$ é o peso seco aferido anteriormente.

$$
A=\frac{P_{h t}-P_{e}}{P_{e}} \times 100
$$

A tabela 1 mostra os resultados obtidos para a absorção de água das fibras no decorrer do tempo em que a mesma permaneceu imersa até o seu ponto de saturação.

Tabela 1: Absorção de água das fibras no tempo.

\begin{tabular}{c|c|c|c|c|c|c|c|c}
\hline Tempo (Horas) & $\mathbf{2 4}$ & $\mathbf{4 8}$ & $\mathbf{7 2}$ & $\mathbf{9 6}$ & $\mathbf{1 2 0}$ & $\mathbf{1 4 4}$ & $\mathbf{1 9 2}$ & $\mathbf{2 4 0}$ \\
\hline Absorção de agua (\%) & 345,72 & 407,34 & 425,8 & 428,7 & 436,98 & 440,2 & 449 & 449 \\
\hline
\end{tabular}

Para a determinação da resistência a tração da fibra, utilizou-se a recomendação da indústria Hysol Grafil [26], uma vez que tal procedimento ainda não é normatizado. Nesse método usasse uma moldura de papel para conter um segmento de fibra e assim com um maior cuidado ancora-la entre as garras da máquina de tração, que nesse caso foi a EMIC DL 10000, com cédula de carga com capacidade máxima de 0,02 kN. Com as leituras obtidas na máquina optou-se por calcular a média aritmética resultando em uma resistência média de 492,62 MPa.

\subsection{Produção dos compósitos}

As argamassas foram inicialmente produzidas a partir de traços usados em outros trabalhos, tais quais [9], [11], [18] a fim de estabelecer comparações. Então se iniciou com o traço de 1:1:0,40 (cimento: areia: relação água/cimento em massa), porém, ao se adicionar as fibras às argamassas, a mistura tornou-se pouco trabalhável, chegando então ao valor de 0,59 para a relação água cimento. Então foram moldados corpos de prova 
prismáticos, de dimensões 50 × 50 x $300 \mathrm{~mm}^{3}$, dos compósitos reforçados com fibra (CRF) com diferentes frações volumétricas e comprimentos de fibras, sendo eles identificados por 2\%CRF25, 3\%CRF25, 2\%CRF45 e 3\%CRF45, em que 2\% e 3\% indicam suas frações volumétricas e 25 e 45 indicam os comprimentos de fibras ( $25 \mathrm{~mm}$ e $45 \mathrm{~mm}$ ). Os corpos de provas de argamassa de referência foram denominados por AR. Os corpos de prova foram mantidos nos moldes por 2 dias em bancada a temperatura e umidade ambiente e em seguida foram imersos em água durante 24 dias, e ficaram por 2 dias no processo de secagem em bancada antes da realização do ensaio de flexão, sendo assim estes foram ensaiados com 28 dias de idade.

\subsection{Ensaio de flexão}

Ensaios de flexão a quatro pontos, com as cargas aplicadas nos terços médios e controladas pelo deslocamento vertical do corpo de prova, foram realizados nos corpos de prova prismáticos de acordo com os procedimentos citados anteriormente. Os ensaios foram realizadas em uma máquina INSTRON, modelo 5500R, com cédula de carga de $5 \mathrm{kN}$ sem o auxílio de extensômetros sendo obtidos os deslocamentos do travessão da máquina. Os ensaios foram realizados em três corpos de prova para cada tipo de compósito e leu-se os resultados para carga máxima $\left(\mathrm{P}_{\max }\right)$ e prolongada $\left(\mathrm{P}_{\text {lop }}\right)$ além do deslocamento no limite de proporcionalidade $\left(\delta_{\mathrm{LOP}}\right)$. Com essas leituras calculou-se o módulo de elasticidade através de equação 3 que é derivada da equação da linha elástica onde P é a carga aplicada, y é o deslocamento dos pontos de aplicação da carga e E é o módulo de elasticidade.

$$
E=\left(6,99 \times 10^{5}\right) \times \frac{P}{y}
$$

A partir das mesmas leituras calculou-se a tensão de flexão no limite de proporcionalidade através de equação 4.

$$
\sigma_{L O P}=2,16 \times 10^{-3} \times P
$$

Com os resultados obtidos calculou-se a média aritmética para a matriz de argamassa de referência e cada tipo de compósito, além da composição das curvas de carga versus deslocamento vertical e através dessas curvas determinou se os índices de tenacidade propostos por cada norma e método citados anteriormente.

\section{RESULTADOS E DISCUSSÃO}

A fibra do curauá teve um papel marcante ao ser incorporada nos compósitos em relação ao desempenho mecânico. A Tabela 2 apresenta os resultados da carga máxima $\left(\mathrm{P}_{\mathrm{MÁx}}\right)$ e prolongada $\left(\mathrm{P}_{\mathrm{LOP}}\right)$, o deslocamento no limite de proporcionalidade $\left(\delta_{\mathrm{LOP}}\right)$, a tensão de limite proporcionalidade $\left(\sigma_{\mathrm{LOP}}\right)$ e o módulo de elasticidade (E) do ensaio de flexão a 4 pontos.

A argamassa de referência (AR) não obteve carga prolongada, sua carga de máxima equivale a carga prolongada acusando sua ruptura, o que não é observado para todas as vigas reforçadas com fibras, a resistência à flexão do compósito é regulada, principalmente por adesão interfacial entre a fibra e a matriz cimentícia [19]. Após a fissuração da matriz os compósitos reforçados com fibras continuaram a suportar carga (Figura 1) além do ponto da carga de pico, apresentando grande deformação.

Tabela 2: Valores médios das propriedades mecânicas obtidas do ensaio de flexão a 4 pontos dos compósitos.

\begin{tabular}{c|c|c|c|c|c}
\hline MATERIAL & $\mathbf{P}_{\text {MÁX }}(\mathbf{N})$ & $\boldsymbol{\delta}_{\text {LOP }}(\mathbf{M M})$ & $\mathbf{P}_{\text {LOP }}(\mathbf{N})$ & $\boldsymbol{\sigma}_{\text {LOP }}(\mathbf{M P a})$ & $\mathbf{E}(\mathbf{G P a})$ \\
\hline AR & $2.092,50$ & 0,69 & $2.092,5$ & 4.52 & 2,12 \\
\hline 2\%CRF25 & $1.965,89$ & 0,94 & $1.470,0$ & 3.18 & 1,46 \\
\hline $3 \%$ CRF25 & $2.424,22$ & 0,93 & $1.830,0$ & 3.95 & 1,37 \\
\hline 2\%CRF45 & $2.212,84$ & 0,62 & $1.855,0$ & 4.01 & 2.07 \\
\hline 3\%CRF45 & $1.686,00$ & 0,65 & $1.387,0$ & 3,00 & 1.48 \\
\hline
\end{tabular}

A característica dos compósitos reforçados com fibras não sofrer ruptura brusca é atribuída principalmente ao fato da alta resistência à tração das fibras e a dependência de sua interação com a matriz [20]. A carga aplicada diretamente na superfície da matriz do compósito é transferida para as fibras mais próximas da superfície, em seguida, continuamente a carga é transferida de fibra a fibra através da matriz e interface [21]. 


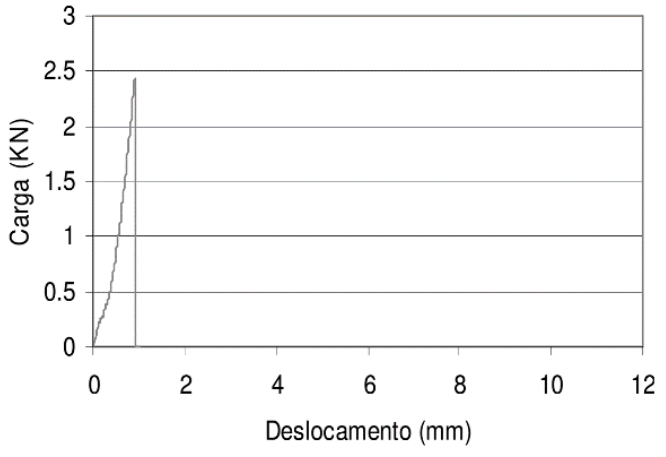

(a)

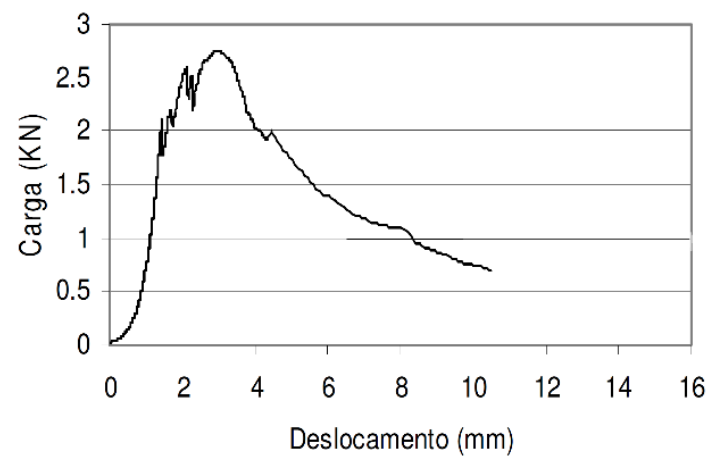

(c)

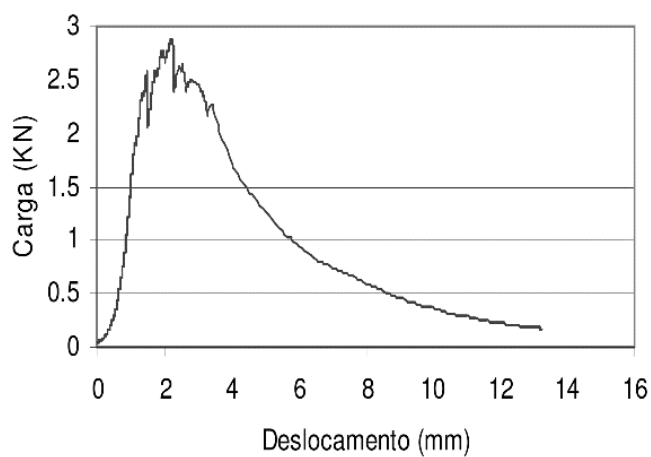

(b)

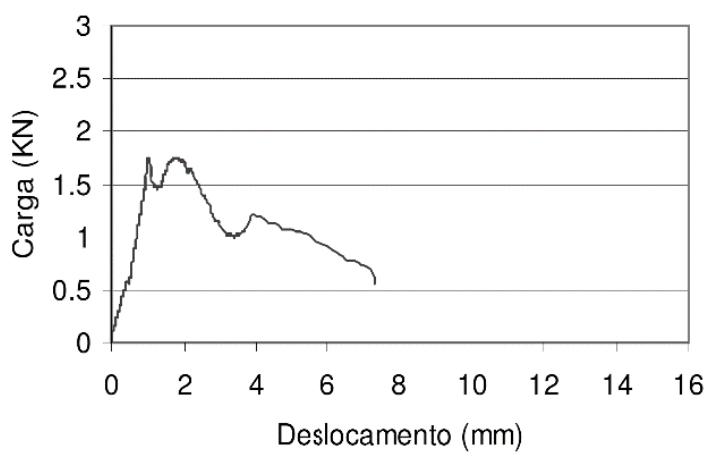

(d)

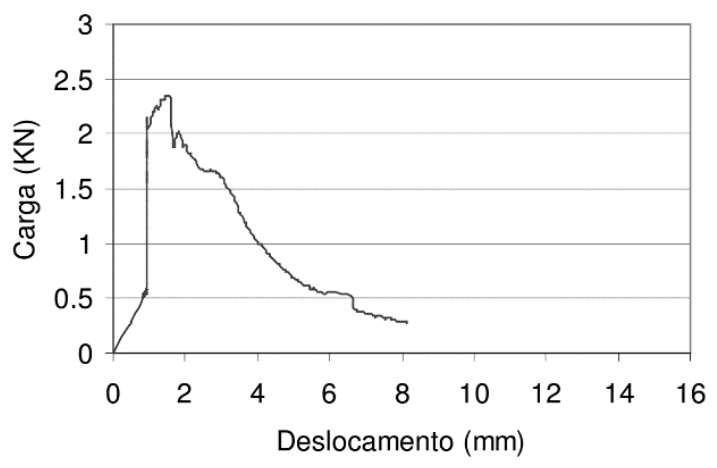

(e)

Figura 1: Curvas típicas carga versus deflexão sob flexão a 4 pontos, dos compósitos (a) AR, (b) 2\%CRF25, (c) 3\%CRF25, (d) 2\%CRF45 e (e) 3\%CRF45.

O aumento a deformação do compósito foi claramente melhorado quando comparada a argamassa de referência. Esse fato pode ser atribuído pela forte adesão interfacial e consequentemente, pela ótima transferência de carga entre a fibra e a matriz [22] levando a alta elasticidade do compósito.

Com o aumento do volume de fibra é observado um aumento na carga máxima. Vale ressaltar que o compósito com maior fração volumétrica e maior comprimento de fibra (3\%CRF45) apresentou um comportamento diferenciando comparado aos demais compósitos reforçado, isso pode ser atribuído a dificuldade de moldagem do mesmo o que influenciou em sua homogeneidade, pois seu comportamento não corresponde ao apresentado pela literatura [23], [24], [25]

Com intenção de caracterizar a tenacidade dos compósitos e comparar o desempenho de diferentes 
frações e comprimentos de fibras, foram determinados, através das curvas de carga por deslocamento, os índices $I_{5}, I_{10}, I_{20}$ e $I_{30}$ propostos pela norma ASTM C1609, Standard test method for flexural performance of Fiber-Reinforced concret (Using Beam with third-point loading) [13] os valores de $\mathrm{F}_{\mathrm{t}} \mathrm{e} \mathrm{T}_{\mathrm{jc}}$, propostos pelo método japonês JSCE -SF4-Method of tests for flexural strength and flexural toughness of steel fiber reinforced concrete [15], além dos índices de resistência e o fator de tenacidade propostos pela norma belga NBN B 15-238 Tests on fibre reinforced concret - Bending test on prismatic sample [14].

Como foi provado anteriormente, através das curvas de carga por deslocamento, os compósitos reforçados com fibras de curauá não apresentam um rompimento brusco como as matrizes de argamassa de referência. Isso se deve às fibras presentes nos compósitos que continuam suportando a carga mesmo após o rompimento da matriz, conferindo assim ductilidade aos compósitos. Tendo isso em vista, não se faz necessário o calculo dos índices de tenacidade para a matriz de argamassa de referência, uma vez que não há qualquer suporte a carga após a ruptura da matriz.

A figura 2 mostra apresenta os índices de tenacidade dos compósitos de acordo com a norma americana ASTM C1609, Standard test method for flexural performance of Fiber-Reinforced concret (Using Beam with third-point loading) [13] Tais índices indicam as áreas absolutas de diferentes partes do gráfico de carga por deslocamento, sendo essa a essência do conceito de tenacidade.

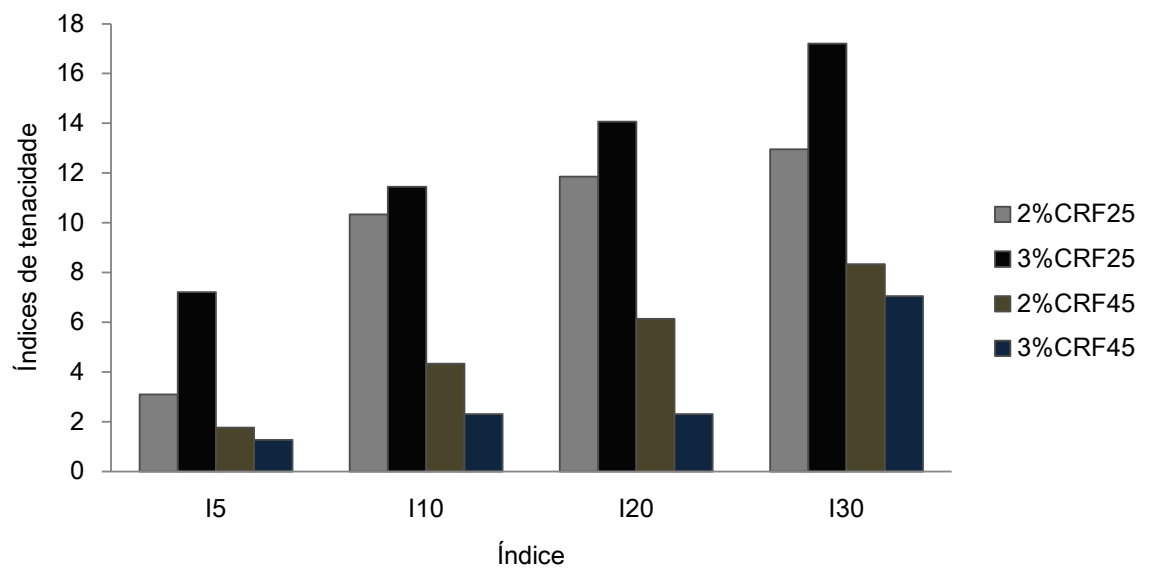

Figura 2: Índices de tenacidade ASTM C1609

A figura 3 apresenta os índices de tenacidade dos compósitos de acordo com o método japonês JSCE -SF4-Method of tests for flexural strength and flexural toughness of steel fiber reinforced concrete [15] em que os valores de $F_{t}$ indicam o equivalente a resistência à tração na flexão e quantificam o trabalho das fibras incorporadas a matriz por meio da tenacidade introduzida ao compósito, e os valores de $\mathrm{T}_{\mathrm{jcl}}$, que mais uma vez indicam a área absoluta do gráfico de carga por deslocamento.

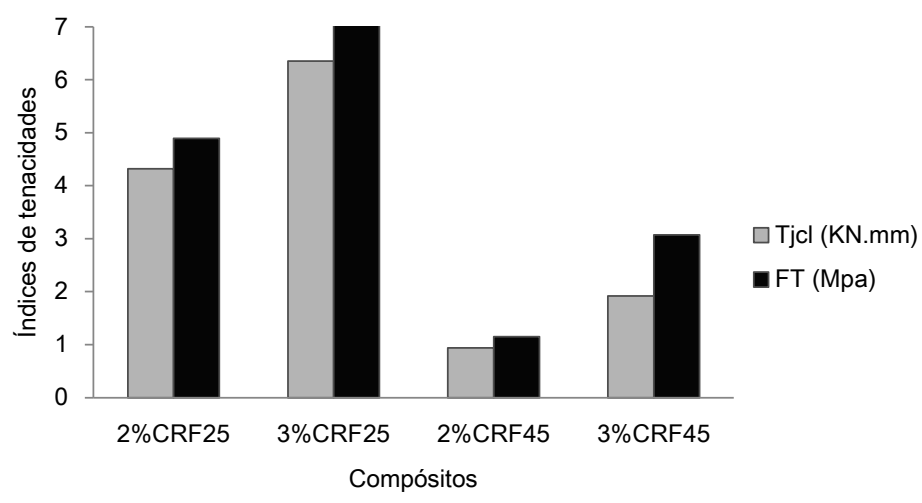

Figura 3: Índices de tenacidade JJCSE - SF4 
As figuras 4 e 5 mostram respectivamente os Índices de resistência $\mathrm{P}_{\mathrm{n}}$, que indicam a razão entre a carga correspondente a fissura e a carga a diferentes deflexões, e de tenacidade $\mathrm{F}_{\mathrm{t}}$, que indicam a área absoluta das curvas de carga por deslocamento, de acordo com a norma belga NBN B 15-238 Tests on fibre reinforced concret - Bending test on prismatic sample [14].

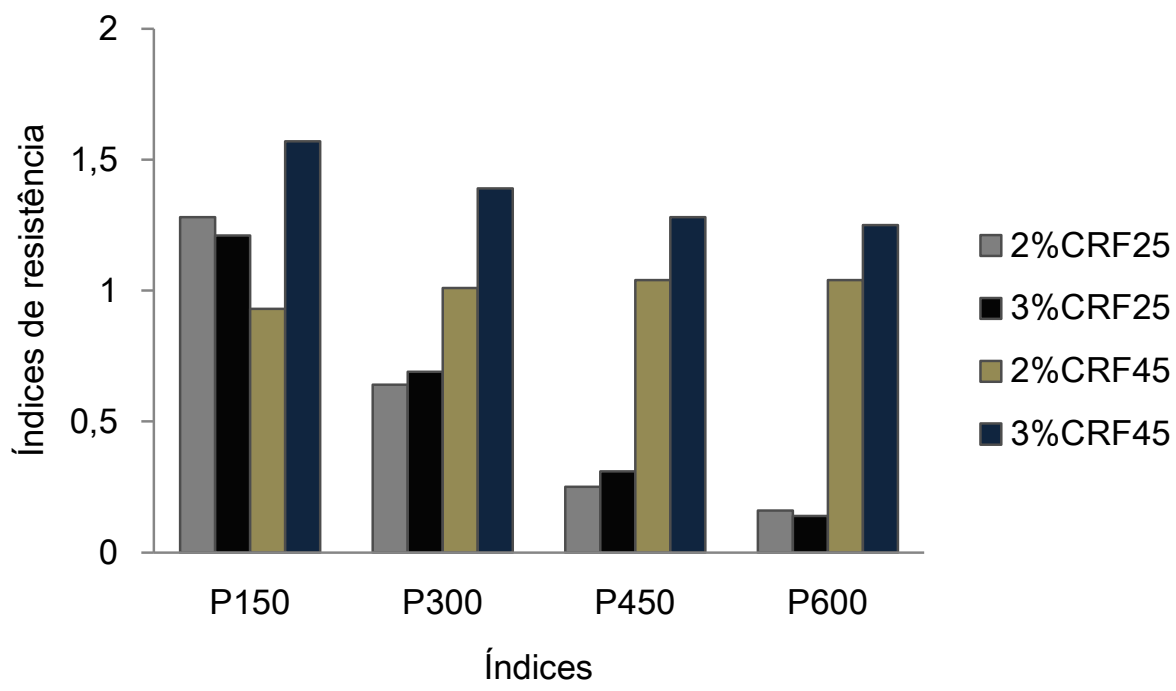

Figura 4: Índices de resistência NBN B15-238

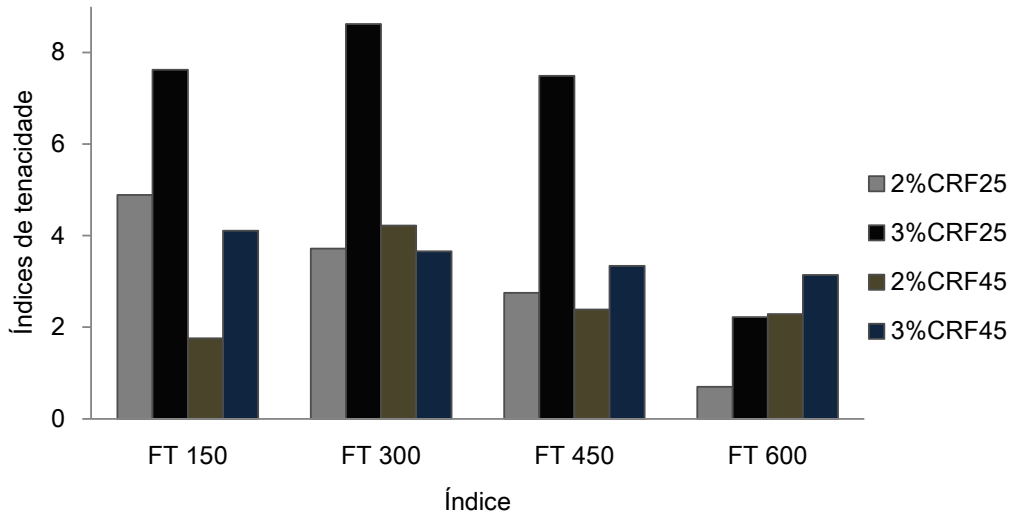

Figura 5: Índices de tenacidade NBN-B15 238

Verificou se que em todos os métodos analisados, os compósitos reforçados com fibras continuaram a suportar parte do carregamento, que pode chegar próximo a $7 \mathrm{MPa}$ no compósito de melhor desempenho. Isso acontece porque as fibras ligam as partes fissuradas após a primeira fissura conferindo coesão aos compósitos.

Pode se analisar o comportamento dos compósitos com o aumento da deflexão através dos índices $\mathrm{P}_{\mathrm{n}}$ em que pode se perceber a capacidade portante residual. Observou se que os compósitos apresentaram um comportamento aproximadamente elasto plástico, com redução da capacidade resistente devido a perda de aderência da fibra com a matriz conforme se aumenta a deflexão.

Com o aumento da resistência pós-fissuração, tem se, por consequência, o aumento da capacidade absorver energia até a ruptura total do compósito, o que constitui o conceito de tenacidade, e assim foi provado através dos índices determinados através dos três diferentes métodos usados neste trabalho.

Para o comportamento dos compósitos após a primeira fissuração, a matriz com maior fração volumétrica e maior comprimento de fibra (3\%CRF45) apresentou melhor desempenho com o aumento da deflexão. Isto deve se ao maior tempo de aderência das fibras com maiores comprimentos com a matriz, uma vez que 
serão necessárias maiores deflexões para ruptura total da matriz, assim os compósitos podem manter a coesão por mais tempo até a ruptura total.

O compósito de maior fração volumétrica e menor comprimento de fibras (3\%CRF45) foi o que apresentou melhor desempenho com relação à tenacidade em todos os métodos analisados, de tal forma que não se observou um padrão no comportamento dos compósitos conforme se variava as frações volumétricas e os comprimentos de fibras. Isto ocorre devido ao procedimento de moldagem dos corpos de provas que tendem a ser mais dificultosos conforme se aumenta as frações volumétricas e os comprimentos de fibra.

\section{CONCLUSÕES}

Foram calculados os índices propostos por três normas apenas para os compósitos, uma vez que a matriz de argamassa de referência não suportou cargas após a primeira fissura. Todos os compósitos apresentaram resistência a flexão após a primeira fissuração e o compósito com maior fração volumétrica e menor comprimento de fibra (3\%CRF25) atingiu o melhor desempenho chegando próximo a $7 \mathrm{MPa}$. Quanto ao comportamento ao longo da deflexão, o compósito com maior fração volumétrica (3\%CRF45) teve o melhor desempenho. Não observou se um padrão no comportamento dos compósitos de diferentes frações volumétricas e comprimentos de fibra, sendo que o compósito 3\%CRF25 também apresentou os melhores índices de tenacidade em todos os métodos utilizados. Assim concluiu se que a inserção de fibras de curauá foi responsável por conferir maior ductilidade ao compósito após a primeira fissuração da matriz. Assim ao invés da fratura brusca apresentada pela matriz de argamassa de referência no início da fissuração, o compósito continua a suportar cargas e absorver energias apresentando grandes deformações.

\section{BIBLIOGRAFIA}

[1] SOUZA, S.F., FERREIRA, M., SAIN, M., et al., "Biofiber Reinforcements in Composite Materials”, In: Woodhead Publishing, pp. 700-720, Cambridge, Reino Unido, 2015.

[2] MONTEIRO, S.N., DEUS J.F., D’ALMEIDA J.R.M. “Mechanical and structural characterization of curaua fibers”, In: Proceedings of characterization of minerals, metals \& materials - TMS Conference, pp. 18. San Antonio, USA, March, 2006.

[3] CARASCHI, J.C., LEÃO, A.L.. "Characterization of curaua fibre”. Molecular Crystals and Liquid Crystals, v. 353, pp. 149-152, Feb. 2001.

[4] MONTEIRO, S.N., LOPES, F.P.D., NASCIMENTO D.C.O., "Processing and properties of contínuos and aligned curaua fibers incorporated polyester composites”, Journal of Materials Research and Technology, v. 2, n. 1, pp. 2-9, Mar. 2013.

[5] GUTIÉRREZ M.C., PAOLI M.A., FELISBERT M.I. “Cellulose acetate and short curuauá fibers biocomposites prepared by large scale processing: Reinforcing and thermal insulating properties”, Industrial Crops and Products, v. 52, pp. 363-271, Jan. 2014.

[6] ARAUJO, J. R., MANO, B., TEIXEIRA, G.M., et al. "Biomicrofibrilar composites of high density polyethylene reinforced with Curauá fibers: Mechanical, interfacial and morphological properties”, Composites Science and Technology, v. 70, n. 11, pp. 1633-1644, Oct. 2010.

[7] CARASCHI, J.C., LEÃO, A.L. Characterization of curaua fibre. Molecular Crystals and Liquid Crystals, In: Repósitorio Institucional UNESP, v.353, pp. 149-152, 2001.

[8] FARUK O., BLEDZKI A.K., FINK F.P., et al. "Biocomposites reinforced with natural fibers: 20002010”, Progress in polymer science, v. 37, n. 11, pp. 1552-1569, Nov. 2011.

[9] RODRIGUES, C. S., Mecânica da Fratura de Compósitos de Matrizes Rígidas - Argamassas Reforçadas com Fibras de Sisal, Dissertação de Mestrado, Departamento de Engenharia Civil Pontifícia, Universaidade Católica do Rio de Janeiro, 1999.

[10] BENTUR, A., MINDESS, S., Fiber Reinforced Cementious Composites, U. K., Elsevier Applied Science, 1990.

[11] TOLEDO F.R D., GHAVAMI K. "Comportamento em Compressão de argamassas reforçadas com fibras naturais”, R. Brás. Eng. Agríc. Ambiental, Campina Grande, v. i, p. 79-88, 1997.

[12] ASSOCIAÇÃO BRASILEIRA DE NORMAS TÉCNICAS, NBR NM 45, Agregados - Determinação da massa unitária e índices de vazios, 8p, Rio de Janeiro, 2006. 
[13] AMERICAN SOCIETY FOR TESTING AND MATERIALS, ASTM C1609, Standard test method for flexural performance of Fiber-Reinforced concret (Using Beam with third-point loading), 9p, Philadelphia, 2010 .

[14] INSTITUT BELGE DE NORMALISATION, NBN B 15-238, Tests on fibre reinforced concret Bending test on prismatic sample, Brussels, 1992.

[15] THE JAPAN SOCIETY OF CIVIL ENGINEERS, JSCE -SF4, Method of tests for flexural strength and flexural toughness of steel fiber reinforced concrete, library of JSCE, Part III-2 Method of tests for steel fiber reinforced concrete, n. 3, pp. 58-61, 1984.

[16] ASSOCIAÇÃO BRASILEIRA DE NORMAS TÉCNICAS, NBR NM 52, Agregado miúdo - determinação da massa específica e da massa específica aparente, 6p, Rio de Janeiro, 2009.

[17] ASSOCIAÇÃO BRASILEIRA DE NORMAS TÉCNICAS, NBR NM 248, Agregado miúdo - determinação da composição granulométrica, 6p, Rio de Janeiro, 2009.

[18] FUJIYAMA R.T., Argamassa de cimento reforçada por fibras de Sisal: Caracterizaçãp mecânica e microestrutural, Tese de M.Sc., Departamento de ciência dos materiais e metalurgia PUC-RIO, Rio de Janeiro, RJ, Brasil, 1997.

[19] TAJVIDI M., FEIZMAND M., FALK R.H., et al. "Effect of cellulose fiber reinforcement on the temperature dependent mechanical performance of nylon 6”, Journal of reinforced plastics and composites, v.28, n. 27, pp. 81-90, 2009.

[20] SEARS K.D., JACOBSON R., CAULFIELD D.F. “Underwood JH. Reinforcement of engineeringthermoplastics with high purity wood cellulose fibers”,In: Sixth International Conference on WoodfiberPalstic Composites, Madison concourse hotel, Madison, Wisconsin, 2001.

[21] DEVI, L.U., BHAGAWAN, S.S., THOMAS, S. "Mechanical properties of pineapple leaf fiber reinforced polyester composites”, Journal of Applied Polymer Science, v. 64, n. 17, pp. 39-48, 1997.

[22]YU, T, REN, J, LI, S, et al. "Effect of fiber surface-treatments on the properties of poly (lactic acid)/ramie composites”, Composites Part A: Applied Science and Manufacturing , v.41, pp.499-505, 2010.

[23] AMINTOWLIEH, Y., SARDASHTI, A., SIMON, L.C. "Polyamide 6-wheat straw composites: Effects of additives on physical and mechanical properties of the composite”, Polymer Composites, v. 12, n. 33, pp. 76-84, 2012.

[24] RAHMAN, R., HUQUE, M., ISLAM, N., "Mechanical properties of polypropylene composites reinforced with chemically treated abaca”, Composites: Part A, v. 40, pp. 511-517, 2009.

[25] RAGOUBI, M., BIENAIME, D., MOLINA, S., et al.“Impact of corona treated hemp fibers on to mechanical properties of propylene composites”, Industrial Crops and Products, v. 31, pp. 344-249, 2010.

[26] HYSOL GRAFIL. "Grafil test methods, section 1 - Fibres, Test reference 101.13, filament tensile strength and modulus”, April, 1977. 